\title{
Estrutura fatorial da escala de auto-imagem: testando modelos alternativos'
}

\author{
Factorial structure of the self-construal scale: \\ testing alternative models
}

Taciano Lemos MILFONT²

\begin{abstract}
Resumo
Desde sua publicação, a Escala de Auto-Imagem tem sido um importante instrumento para medir as auto-imagens Independente e Interdependente. No Brasil, a Escala de Auto-Imagem foi adaptada por Gouveia, Singelis e Coelho (2002), que confirmaram sua estrutura bifatorial. O estudo de Hardin, Leong e Bhagwat (2004), no entanto, aponta uma estrutura multifatorial, com seis fatores subjacentes aos itens da Escala de Auto-Imagem. Este estudo apresenta resultados de análises fatoriais confirmatórias com base em dados de 658 universitários, a maioria do sexo masculino e com idades variando entre 17 e 42 anos ( $M=19,9$; $\mathrm{DP}=2,5)$. O modelo hierárquico, com seis fatores de primeira ordem e dois de segunda, apresentou índices satisfatórios de bondade de ajuste $\left[\chi^{2}(398)=1163,99\right.$; índice de bondade de ajuste=0,89; índice de bondade de ajuste ajustado=0,88; raiz quadrada média do erro de aproximação=0,054; raiz quadrada média residual estandartizada=0,064]. Resultados de correlações com os valores humanos básicos e diferenças quanto ao gênero também são apresentados, confirmando a validade dos seis novos fatores.
\end{abstract}

Palavras-chave: análise fatorial; auto-imagem; escala de auto-imagem; estudantes universitários; interdependência (personalidade).

\begin{abstract}
Since its publication, the Self-Construal Scale has been an important instrument in self-construal measuring. The Self-Construal Scale was adapted to a Brazilian population by Gouveia, Singelis and Coelho (2002), who confirmed its two-factor structure (i.e., Independent and Interdependent Self-Construal). Recently, however, six factors underlying items on the Self-Construal Scale were found (Hardin, Leong \& Bhagwat, 2004). The present study reports confirmatory factor analyses of data from 658 Brazilian undergraduate students, most of them males and with ages ranging from 17 to 42 years old (19.9 2.5 ). A hierarchical model, with six first-order and two second-order factors, presented good fit for the data $\left[\chi^{2}(398)=1163.99\right.$; goodness-of-fit index $=.89$; ajusted goodness-of-fit index $=.88$; root mean square error of approximation $=.054$; standardized root mean square residual $=.064]$. Results also provide support for the usefulness of these new factors in exploring correlation with basic human values and gender differences.
\end{abstract}

Key words: factor analysis; selfconcept; self-construal scale; college students; interdependence (personality).

O estudo do selfé um tema central nas Ciências Humanas e Sociais (Kuhn \& McPartland, 1954).
Pesquisadores interessados nesse construto buscam diferenciar self e ego. Uma das mais freqüentes

\section{v V V V}

- Artigo elaborado a partir da dissertação de T.L. MILFONT, intitulada "A intenção de constituir família: suas bases normativas e relacionais". Universidade Federal da Paraíba, 2001

2 Professor Assistente da Universidade Federal de Alagoas, Maceió, AL, Brasil. Endereço para correspondência: Department of Psychology, The University of Private Bag 92019, Auckland, New Zealand.E-mail:<milfont@gmail.com>, <t.milfont@auckland.ac.nz>.

Agradecimentos: a CAPES, a Valdiney V. Gouveia e a Jorge Artur P. M. Coelho. 
diferenciações feitas é a atribuição de função perceptiva e de função ativa, que ora são atribuídas a apenas um dos construtos (função perceptiva para o self e função ativa para o ego, e vice-versa), ora consideradas supérfluas (Tamayo, 1981). Neste trabalho foi levada em consideração a distinção feita por Asch (1952/1972) entre esses dois conceitos. Segundo ele, o ego é a parte organizada do indivíduo que representa o organismo ou a reação do organismo a suas próprias ações, e o self seria o representante consciente do ego. Assim, "o eu é a representação fenomenal do ego, o ego tornado consciente" (Asch, 1952/1972, p. 239). Vale ressaltar que o conceito de "eu" proposto por Asch tem o mesmo sentido de self, tratando-se apenas de uma tradução para o português, da mesma forma que autores tratam esse construto como yo no contexto espanhol (Gouveia \&Clemente, 1998; Espinosa, 2000).

Nas últimas décadas, o estudo do self tem despertado um renovado interesse em Psicologia Social e Psicologia Transcultural (Kagitçibasi, 1997), principalmente a partir dos estudos sobre individualismo-coletivismo (Triandis, 1995). Embora o conceito de self tenha sempre estado presente em estudos comparativos entre culturas individualistas e coletivistas (Smith \& Bond, 1993; Kagitçibasi, 1997), foi a partir do trabalho de Markus e Kitayama (1991) sobre a construção do self que esses estudos foram mais enfatizados. Esses autores propuseram uma visão dual do self, apresentando-o como independente e interdependente, e posteriormente foi desenvolvida uma medida para avaliar essas duas dimensões (Singelis, 1994). Tal medida foi adaptada para o contexto brasileiro (Gouveia, Singelis \& Coelho, 2002b), todavia uma revisão da sua estrutura fatorial foi recentemente apresentada (Hardin, Leong \& Bhagwat, 2004). Este estudo tenta contribuir para a validação dessa medida no Brasil, considerando esta nova estrutura fatorial apresentada. Antes, porém, faz-se necessário um maior aprofundamento na conceituação do self dual e nos estudos prévios que tratam da estrutura fatorial da medida.

\section{O modelo de self dual}

Para Markus e Kitayama (1991), a construção do selfé um fenômeno importante para o entendimento de processos psicológicos (cognição, emoção e motivação) que apresentam variações entre culturas, propondo duas esferas: independente e interdependente. Embora tratadas em inglês como self-construals (Markus \& Kitayama, 1991; Singelis, 1994) e em espanhol como construcciones del yo (Gouveia \& Clemente, 1998; Espinosa, 2000), esses construtos vêm sendo referidos no Brasil como auto-imagens (Gouveia, Coelho \& Vasconcelos, 1999; Gouveia et al., 2002b; Milfont, Gouveia, Chaves, Socorro \& Queiroga, 2002), e serão desta forma abordados neste estudo. A auto-imagem indica como as pessoas vêem a si mesmas, se separadas (auto-imagem independente) ou conectadas (auto-imagem interdependente) às demais.

Para esse modelo, o self não é assumido como completo, um todo, uma entidade autônoma, sem os outros. Os outros e o social são importantes para ambas as auto-imagens, mas para cada uma a relação se dá de forma diferenciada (Figura 1A e 1B). Para a auto-imagem independente, o self é caracterizado como estável, unitário e delimitado. Quando pensam sobre si mesmos, indivíduos com esse tipo de construção do selfaltamente desenvolvido tomam como referência suas próprias habilidades, características, atributos e objetivos, em lugar dos pensamentos, sentimentos e ações dos outros. Já quando pensam sobre os demais, consideram características individuais de cada pessoa em lugar de fatores relacionais ou contextuais. A auto-imagem interdependente, em contraste, define-se como flexível e variável. Os indivíduos com esse tipo de self não se concebem separados da situação em que se encontram, mas sim modelados por ela. Dependem dos demais e de suas relações com eles, regulando seus comportamentos sobre fatores contextuais (Markus \& Kitayama, 1991; Singelis, 1994).

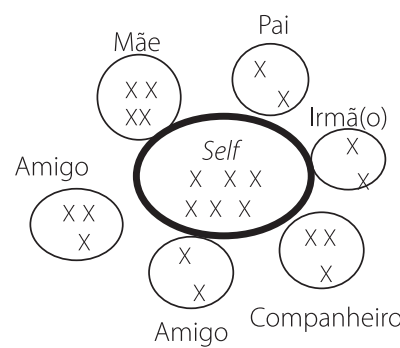

A

Auto-Imagem Independente

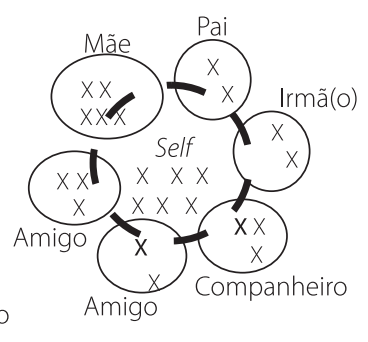

B

Auto-Imagem Interdependente
Figura 1. Representação conceitual das auto-imagens (adaptado de Markus \& Kitayama, 1991). 


\section{A escala de auto-imagem independente e interdependente}

A Escala de Auto-Imagem (EAI) tem o objetivo de medir o conjunto de pensamentos, sentimentos e ações que compõem as auto-imagens independente e interdependente. Essa escala completou uma década de existência (Singelis, 1994) e tem sido amplamente utilizada. Por exemplo, essa medida aparece mais de 180 vezes no Social Science Citation Index. Além disso, uma ou as duas subescalas já foram utilizadas em mais de 50 estudos, traduzidas para diversas línguas (Árabe, Chinês, Espanhol, Hebreu, Japonês e Português), e administradas em sete países (Hardin et al., 2004).

Pesquisas têm demonstrado, ainda, que o construto de self dual pode ser utilizado em amplos aspectos das relações sociais, pois os fatores que caracterizam a auto-imagem das pessoas, se conectadas ou separadas das demais, possuem poder explicativo em âmbitos diversos (Markus \& Kitayama, 1991). Assim, esse construto tem sido utilizado na explicação de diferentes variáveis, tais como: a intenção de constituir família (Milfont, Gouveia \& Costa, no prelo), atitudes de ação afirmativa (Ozawa, Crosby \& Crosby, 1996), maturidade de carreira (Hardin, Leong \& Osipow, 2001), processo de comunicação (Singelis \& Brown, 1995), autoestima (Sato \& Cameron, 1999), sentimento de constrangimento (Sharkey \& Singelis, 1995; Singelis \& Sharkey, 1995; Singelis, Bond, Sharkey \& Lai, 1999; Gouveia, Singelis, Guerra, Santos \& Vasconcelos, 2004), moralidade (Espinosa, 2000; Cohen \& Rozin, 2001), biculturalismo (Yamada \& Singelis, 1999), e relações interpessoais (Gouveia, Coelho \& Vasconcelos, 1999). Além das suas relações com os valores humanos (Oishi, Schimmack, Diener \& Suh, 1998; Milfont et al., 2002) e das suas diferenças quanto ao gênero (Cross \& Madson, 1997; Hardin et al., 2004).

\section{A estrutura fatorial da EAI}

Elaborada por Singelis (1994) com amostra estadunidense, essa escala obteve alfa de Cronbach $(\boldsymbol{\alpha})$ de 0,70 e 0,74 para as subescalas de auto-imagem independente e interdependente, respectivamente. A versão inicial da escala é composta por 24 itens, embora seis novos itens também tenham sido apresentados. Singelis (1994) utilizou Análises dos Componentes
Principais (ACP) e Análises Fatoriais Confirmatórias (AFC) para demonstrar a validade de construto da EAl e sua bidimensionalidade. Esse autor demonstrou, ainda, a validade preditiva da escala (para uma descrição mais aprofundada, ver Gouveia et al., 2002a).

A EAI foi adaptada e validada para o contexto brasileiro recentemente por Gouveia et al. (2002a), que realizaram um estudo com 400 alunos do ensino médio, em sua maioria do sexo feminino $(67,3 \%)$ e com idade média de 20 anos ( $D P=5,60)$. A versão com 30 itens foi utilizada, mas apenas os 24 itens da versão inicial foram considerados. Resultados de ACP e AFC indicaram que os 24 itens da escala, eqüitativamente distribuídos entre as auto-imagens independente e interdependente, formaram as duas dimensões propostas. Especificamente, verificaram que a solução na qual as duas dimensões se correlacionaram livremente foi a que apresentou a melhor bondade de ajuste, contrariando uma estrutura ortogonal sugerida por Singelis (1994). As escalas apresentaram índices de consistência interna ( $\alpha$ ) meritórios no contexto brasileiro: 0,54 para a auto-imagem interdependente e 0,52 para a independente (Gouveia et al., 2002a).

\section{Uma estrutura fatorial alternativa}

Embora reconheçam a importância da EAl e os resultados psicométricos apresentados por Singelis (1994), Hardin et al. (2004) sugerem que novos estudos sejam realizados para que a estrutura fatorial latente do construto dual de self possa ser conhecida. Eles apontam três motivos principais. Inicialmente, argumentam que métodos estatísticos mais apropriados devem ser utilizados. Singelis (1994) no estudo de validação utilizou ACP para verificar a estrutura fatorial. Hardin e cols. argumentam que a Análise Fatorial Exploratória (AFE) é o método mais apropriado para a identificação de fatores latentes (Fabrigar, Wegener, MacCallum \& Strahan, 1999; Laros, 2004).

Em segundo lugar, esses autores verificaram que embora exista uma versão com 30 itens, apenas poucos estudos incluíram alguns dos novos itens em ACP, mas nenhum estudo considerou os seis novos itens em uma AFE. Por fim, eles argumentam que novos estudos devem ser realizados, pois pesquisas sobre individualismo-coletivismo têm verificado tratarem-se de 
construtos multidimensionais (Triandis, 1995; Singelis, Triandis, Bhawuk \& Gelfrand, 1995; Gouveia, Andrade, Jesus, Meira \& Soares, 2002b). Eles concluem que a melhor forma de medir o construto dual de self é através de melhores e mais refinadas distinções dentro de cada uma das duas dimensões mais gerais, isto é, das autoimagens independente e interdependente.

Para testar suas idéias, Hardin et al. (2004) realizaram um estudo com 786 universitários estadunidenses de dois contextos étnico-raciais (estadunidenses asiáticos e caucasianos) que responderam a EAI. Os participantes foram eqüitativamente divididos em dois grupos. Ambos os grupos foram compostos em sua maioria por mulheres (55,2\% e 53,4\% para Grupo 1 e 2, respectivamente), e tiveram idade média de 19 anos ( $\mathrm{DP}=2,52$ e 2,58 para Grupo 1 e 2 , respectivamente). Inicialmente foi testada a estrutura bifatorial proposta por Singelis (1994), utilizando-se os 30 itens da escala. Resultados de AFE e AFC indicaram que a estrutura bifatorial foi significantemente melhor do que o modelo unifatorial nos dois grupos, embora os índices de bondade de ajuste não tenham apresentado um adequado ajuste aos dados. Os índices de consistência interna foram 0,69 para a auto-imagem independente e 0,68 para a interdependente. Verificaram ainda que alguns itens interdependentes também apresentaram saturações no fator independente: os itens 03 e 30 apresentaram saturações negativas, enquanto que o item 06 apresentou saturação positiva.

Após essa primeira análise, eles realizaram outra AFE com o Grupo 1 testando a possibilidade da emergência de mais fatores. Baseados em índices de adequação, eles fixaram a extração de seis fatores, que foram denominados de Autonomia/Assertividade $(\alpha=0,59)$, Individualismo $(\alpha=0,64)$, Consistência Comportamental $(\alpha=0,61)$, Primazia do Eu $(\alpha=0,43)$, Estima pelo Grupo $(\alpha=0,60)$ e Interdependência Relacional $(\alpha=0,52)$. Os quatro primeiros fatores foram compostos por 16 itens da dimensão Independente, com exceção de dois itens Interdependentes (06 e 03)

$\rightarrow \quad$ que saturaram nos fatores Individualismo e Autono3 mia/Assertividade, respectivamente. Os outros dois 寉 fatores foram compostos unicamente por itens $\sum_{-1}$ Interdependentes. Assim, enquanto os primeiros quatro fatores representam tipos de auto-imagem Independente, os dois últimos indicam tipos de auto-imagem Interdependente.
A validade de construto dos seis fatores foi testada com um modelo hierárquico utilizando o Grupo 2. Esse modelo apresentou adequados índices de bondade de ajuste, confirmando a possibilidade dos seis fatores. Para examinar a utilidade desses fatores, Hardin et al. (2004) realizaram uma Manova para verificar diferenças quanto ao sexo e etnia em relação aos dois fatores originais da EAl e aos seis novos fatores descritos acima. Os resultados indicaram que os dois grupos étnicos (asiáticos e caucasianos) diferem em alguns, mas não em todos os seis novos fatores. O mesmo foi verificado com relação ao sexo. Embora não tenham sido encontradas diferenças significativas em relação aos fatores Independente e Interdependente, homens apresentaram escores estatisticamente superiores aos das mulheres no fator Interdependência Relacional. Em conjunto, esses resultados suportam a estrutura fatorial alternativa, com seis fatores latentes aos itens da EAI.

A partir dos resultados descritos acima, os objetivos do presente estudo foram: (1) investigar a validade de construto da EAl, no contexto brasileiro, com uma amostra diferente da utilizada na sua validação (Gouveia et al., 2002a), incluindo, ainda, os seis novos itens; (2) testar o modelo fatorial alternativo com seis fatores; e (3) confirmar a utilidade do modelo alternativo (validade de critério e convergente-discriminante).

A validade de construto foi avaliada por meio de análises fatoriais confirmatórias e consistência interna (alfa de Cronbach). A validade de critério foi examinada pelas diferenças dos escores das escalas quanto ao gênero. Pesquisas têm indicado que as mulheres tendem a apresentar uma auto-imagem Independente, enquanto que os homens uma auto-imagem Independente (Cross \& Madson, 1997). Com relação à estrutura fatorial alternativa, Hardin et al. (2004) verificaram que os homens apresentaram escores superiores aos das mulheres no fator Interdependência Relacional.

Finalmente, a validade convergente-discriminante foi verificada por meio de correlações entre as duas auto-imagens e os valores humanos básicos. Estudos prévios verificaram correlações diretas entre os valores humanos que atendem a uma orientação Pessoal e Social com as auto-imagens Independente e Interdependente, respectivamente (Oishi et al., 1998; Milfont et al., 2002). 


\section{Método}

Participaram da pesquisa 658 estudantes universitários de Recife, $\mathrm{PE}$, com idades variando entre 17 e 42 anos ( $M=19,9 ; D P=2,5)$, e em sua maioria do sexo masculino $(51,2 \%, n=227)$. Para a realização das validações cruzadas (cross-validations), a amostra foi aleatoriamente dividida em dois grupos, denominados Grupo 1 e Grupo 2. O Grupo 1 ( $n=327$ ) foi composto em sua maioria por homens (56,0\%; $n=183$ ) com idades entre 17 e 42 anos ( $M=19,8 ; D P=2,6)$, e o Grupo 2 ( $n=331)$ foi composto em sua maioria por mulheres (53,5\%; $n=177)$, com idades entre 17 e 31 anos ( $M=19,9 ; D P=1,5)$.

Os participantes responderam a um questionário composto por cinco partes (Milfont, 2001), mas apenas duas serão tratadas aqui.

- Escala de Auto-Imagem (EAI): Foi utilizada a versão completa com 30 itens, igualmente distribuídos entre as duas auto-imagens: Independente (por exemplo: "Gosto de ser único e diferente das outras pessoas em muitos aspectos"; "Faço minhas próprias coisas, independentemente do que pensam as outras pessoas"), e Interdependente (por exemplo: "Sacrificarei meu próprio interesse em benefício do grupo em que estou"; "Sinto que meu destino se mistura com o destino daqueles que me rodeiam"). Todos os itens da escala apresentam a mesma direção e os respondentes expressam seu grau de concordância em uma escala de sete pontos, tipo Likert, de 1 (Discordo Fortemente) a 7 (Concordo Fortemente).

- Questionário dos Valores Básicos: Este instrumento, proposto por Gouveia (1998), é composto por 24 valores básicos. Todos os valores são terminais, expressando um substantivo. Segundo Gouveia (1998), os 24 valores básicos se organizam em três grandes grupos, segundo o critério de orientação que a pessoa adota: Pessoal, Central ou Social. Esses critérios de orientação se subdividem, por sua vez, segundo a função psicossocial dos valores específicos: experimentação e realização (Pessoal); suprapessoal e existência (Central); e interacional e normativo (Social). A validade e precisão desse modelo têm sido demonstradas em diversos estudos. A estrutura dos valores básicos foi comprovada, por exemplo, no Brasil e na Espanha com universitários
(Gouveia, 1998), e com crianças no Brasil (Maia, 2000). Gouveia (2003a) também apresentou evidências da compatibilidade desse modelo com a principal tipologia dos valores humanos, desenvolvida por Schwartz (1994). Os respondentes expressam o grau de importância de cada valor básico como princípio-guia em suas vidas por meio de uma escala de sete pontos, indo de 1 (Nada Importante) a 7 (Muito Importante). No presente estudo apenas os três critérios de orientação foram considerados. Esses critérios de orientação, com os seus respectivos valores, são: Pessoal (emoção, sexual, prazer, estimulação, êxito, poder, prestígio, autodireção e privacidade), Central (justiça social, beleza, maturidade, sabedoria ${ }^{3}$, saúde, estabilidade pessoal e sobrevivência) e Social (convivência, honestidade, apoio social, afetividade, religiosidade, obediência, tradição e ordem social).

Os dados foram coletados no segundo semestre de 2000, após autorização prévia das universidades. As escalas foram administradas intercaladamente, procurando evitar o efeito de exposição da informação e possível viés de resposta. Inicialmente, foram esclarecidos os objetivos da pesquisa, garantindo aos participantes o anonimato. Os questionários foram respondidos individualmente pelos universitários, numa média de 20min, em situação coletiva em sala de aula.

Foram utilizados os pacotes estatísticos SPSSWIN, versão 12.0.1, e LISREL, versão 8.54. Antes da análise dos dados, a variância e normalidade dos itens foram checadas. Nenhum dos itens da EAl teve variância perto de zero, indo de 0,56 a 3,96 ( $M=2,46$; $\mathrm{DP}=0,17)$. A normalidade dos itens foi investigada em termos dos valores de skewness (indo de -1,91 a 0,80, $|M|=.55$ ) e kurtosis (indo de $-1,43$ a 5,48, $|M|=0,28$ ). Esses valores estão abaixo do recomendado para AFC utilizando o estimador Máxima Verossimilhança (Maximum Likelihood, ML; skewness $<2$, kurtosis $<7$, West, Finch \& Curran, 1995), indicando a normalidade dos itens. A técnica de Imputação Múltipla (Multiple Imputation) (Jöreskog \& Sörbom, 1996; Schafer, 1997) foi utilizada para substituir os casos em branco. Essa técnica é correntemente implementada em Psicologia (Sinharay, Stern \& Russell, 2001; Schafer \& Graham, 2002). 
Existem diversos índices de bondade de ajuste no âmbito das AFC utilizando os Modelos de Equação Estrutural (Structural Equation Models, SEM), por isso aconselha-se o uso de múltiplos indicadores (Thompson, 2000). No presente estudo, foram utilizados os seguintes índices de bondade de ajuste: a razão do qui-quadrado pelo grau de liberdade ( $\chi^{2} / g$.I.), o índice de bondade de ajuste (Goodness-of-Fit Index, GFI), o índice de bondade de ajuste ajustado (Ajusted Goodnessof-Fit Index, AGFI), a raiz quadrada média do erro de aproximação (Root Mean Square Error of Approximation, RMSEA) com o seu intervalo de confiança de 90\% (90 Percent Confidence Interval for RMSEA), e a raiz quadrada média residual estandardizada (Standardized Root Mean Square Residual, SRMR).

Valores do $\chi^{2} /$ g.l. inferiores a 5,00 podem ser interpretados como uma aceitável adequação do modelo, mas se recomendam valores entre 2,00 e 3,00 (Carmines \& Mclver, 1981). Para a verificação da adequação do modelo aos dados são recomendados, ainda, valores de GFI e AGFI superiores a 0,90 (Garson, 2003), mas valores a partir de 0,80 já indicam modelos aceitáveis (Rhee, Uleman, \& Lee, 1996). Com relação aos valores de RMSEA e SRMR são recomendados valores próximos a 0,06 e 0,08, respectivamente (Hu \& Bentler, 1999).

Para a seleção de modelos concorrentes, mais três indicadores foram utilizados, além da análise do melhoramento nos outros índices (Hong, Malik \& Lee, 2003). Os três indicadores foram: o teste da diferença do qui-quadrado (Chi-Square Difference Test), o índice esperado de validação cruzada (Expected Cross-Validation Index, ECVI) com o seu intervalo de confiança de $90 \%$ (90 Percent Confidence Interval for ECVI) e o critério de informação consistente de Akaike (Consistent Akaike Information Criterion, CAIC). Resultados estatisticamente significativos no teste da diferença do qui-quadrado, e menores valores no ECVI e CAIC indicam o modelo que melhor se ajusta aos dados (Garson, 2003). validade de construto da EAI proposta por Singelis (1994)
e encontrada por Gouveia et al. (2002a), utilizando-se a escala completa. Assim, AFC (método de Máxima
Verossimilhança), utilizando a matriz de covariância dos 30 itens da escala, foi realizada com o Grupo 1. Inicialmente dois modelos foram testados. No Modelo 1, unifatorial, todos os itens foram especificados para apresentarem saturação em um único fator. No Modelo 2, bifatorial, os 15 itens que teoricamente representam a auto-imagem Independente foram especificados para saturarem no fator correspondente, o mesmo sendo feito com os outros 15 itens correspondentes à Interdependente.

Como pode ser visto (Tabela 1), o modelo bifatorial (Modelo 2) apresentou melhores índices de bondade de ajuste do que o unifatorial (Modelo 1), sendo essa diferença estatisticamente significativa $\left[\Delta \chi^{2}(1)=181,41, p<0,001\right]$. Esses resultados corroboraram a estrutura fatorial proposta por Singelis (1994) e verificada, no Brasil, por Gouveia et al. (2002a) quando utilizaram apenas 24 itens. Os fatores apresentaram uma pequena correlação positiva mas não foi estatisticamente significativa $(\phi=0,10, p>0,05)$. As subescalas Independente e Interdependente apresentaram alfa de Cronbach de 0,50 e 0,54, respectivamente.

A partir da confirmação da estrutura bifatorial, outros três modelos foram testados para verificar a ortogonalidade dos fatores. A correlação dos dois fatores ( $\phi$ ) foi especificada em -1,00 (Modelo 3), 0,00 (Modelo 4) e +1,00 (Modelo 5). Os Modelos 3 e 5 apresentaram resultados idênticos aos do modelo unifatorial (Modelo 1), e, assim, também apresentaram resultados estatisticamente inferiores ao modelo de estimação livre (Modelo 2) $\left[\Delta \chi^{2}(1)=181,41, p<0,001\right]$ (Tabela 1).

Apenas os Modelos 2 e 4 apresentaram índices satisfatórios. $\mathrm{O}$ modelo de estimação livre (Modelo 2) apresentou uma pequena redução no ECVI e foi marginalmente superior ao modelo ortogonal (Modelo 4) $\left[\Delta \chi^{2}(1)=3,39, p<0,07\right]$. Esses modelos foram testados com o Grupo 2. Como pode ser visto (Tabela 1), os índices foram bastante similares. Para esse grupo, o modelo de estimação livre (Modelo 7) foi estatisticamente superior ao ortogonal (Modelo 8) $\left[\Delta \chi^{2}(1)=3,39, p<0,05\right]$.

Embora o modelo de estimação livre tenha apresentado os melhores índices de bondade de ajuste para ambos os grupos, Modelo 2 para o Grupo 1 e Modelo 7 para o Grupo 2, alguns índices de modificação foram significativos, indicando a presença de alguns 
Tabela 1. Índices de bondade de ajuste dos modelos alternativos da Escala de Auto-Imagem (EAI).

\begin{tabular}{|c|c|c|c|c|c|c|c|c|c|}
\hline Modelo & $\chi^{2}$ & g.l. & $\chi^{2} / g .1$. & $\mathrm{GFI}$ & AGFI & CAIC & ECVI $(90 \%$ IC) & RMSEA $(90 \%$ IC) & SRMR \\
\hline \multicolumn{10}{|l|}{ Grupo $1(n=327)$} \\
\hline Modelo 1. Unifatorial & 1159,66 & 405 & 2,86 & 0,81 & 0,78 & 1567,05 & $\begin{array}{c}3,93 \\
(3,62 ; 4,25)\end{array}$ & $\begin{array}{c}0,076 \\
(0,071 ; 0,081)\end{array}$ & 0,082 \\
\hline Modelo 2. Bifatorial livre & 978,25 & 404 & 2,42 & 0,83 & 0,81 & 1392,44 & $\begin{array}{c}3,37 \\
(3,11 ; 3,67)\end{array}$ & $\begin{array}{c}0,066 \\
(0,061 ; 0,071)\end{array}$ & 0,079 \\
\hline $\begin{array}{l}\text { Modelo 3. Correlação fixada } \\
\text { em }-1,0\end{array}$ & 1159,66 & 405 & 2,86 & 0,81 & 0,78 & 1567,05 & $\begin{array}{c}3,93 \\
(3,62 ; 4,25)\end{array}$ & $\begin{array}{c}0,076 \\
(0,071 ; 0,081)\end{array}$ & 0,082 \\
\hline $\begin{array}{l}\text { Modelo 4. Correlação fixada } \\
\text { em } 0,0\end{array}$ & 981,64 & 405 & 2,42 & 0,83 & 0,81 & 1389,04 & $\begin{array}{c}3,38 \\
(3,11 ; 3,67)\end{array}$ & $\begin{array}{c}0,066 \\
(0,061 ; 0,071)\end{array}$ & 0,079 \\
\hline $\begin{array}{l}\text { Modelo 5. Correlação fixada } \\
\text { em }+1,0\end{array}$ & 1159,66 & 405 & 2,86 & 0,81 & 0,78 & 1567,05 & $\begin{array}{c}3,93 \\
(3,62 ; 4,25)\end{array}$ & $\begin{array}{c}0,076 \\
(0,071 ; 0,081)\end{array}$ & 0,082 \\
\hline $\begin{array}{l}\text { Modelo 6. Bifatorial livre com } 6 \\
\text { itens saturando nos } 2 \text { fatores }\end{array}$ & 869,99 & 398 & 2,18 & 0,85 & 0,82 & 1324,92 & $\begin{array}{c}3,08 \\
(2,83 ; 3,35)\end{array}$ & $\begin{array}{c}0,060 \\
(0,055 ; 0,066)\end{array}$ & 0,071 \\
\hline \multicolumn{10}{|l|}{ Grupo $2(n=331)$} \\
\hline Modelo 7. Bifatorial livre & 933,57 & 404 & 2,31 & 0,84 & 0,82 & 1348,50 & $\begin{array}{c}3,20 \\
(2,94 ; 3,48)\end{array}$ & $\begin{array}{c}0,063 \\
(0,058 ; 0,068)\end{array}$ & 0,073 \\
\hline $\begin{array}{l}\text { Modelo 8. Correlação fixada } \\
\text { em } 0,0\end{array}$ & 939,11 & 405 & 2,31 & 0,84 & 0,82 & 1347,24 & $\begin{array}{c}3,21 \\
(2,95 ; 3,49)\end{array}$ & $\begin{array}{c}0,063 \\
(0,058 ; 0,069)\end{array}$ & 0,074 \\
\hline $\begin{array}{l}\text { Modelo 9. Bifatorial livre com } 6 \\
\text { itens saturando nos } 2 \text { fatores }\end{array}$ & 906,00 & 398 & 2,27 & 0,85 & 0,82 & 1361,75 & $\begin{array}{c}3,15 \\
(2,90 ; 3,43)\end{array}$ & $\begin{array}{c}0,062 \\
(0,057 ; 0,068)\end{array}$ & 0,071 \\
\hline
\end{tabular}

$\chi^{2}=$ qui-quadrado; g.l.= graus de liberdade; $\chi^{2} / \mathrm{g} . \mathrm{I}=$ = razão do qui-quadrado pelo grau de liberdade; GFI= índice de bondade de ajuste; AGFI= índice de bondade de ajuste ajustado; $C A I C=$ critério de informação consistente de Akaike; $E C V I=$ índice esperado de validação cruzada; $90 \% \mid C=$ intervalo de consistência de $90 \%$ do ECVI; RMSEA = raiz quadrada média do erro de aproximação; $90 \% \mid C=$ intervalo de consistência de $90 \%$ do RMSEA; SRMR= raiz quadrada média residual estandartizada.

itens na outra dimensão. Assim, outro modelo (Modelo 6) foi proposto para o Grupo 1: alguns itens $(01,17,24$, 28, 29 e 30) foram especificados para saturarem em ambos os fatores. Como pode ser visto na Tabela 1, esse modelo foi o que apresentou os melhores índices de bondade de ajuste, sendo estatisticamente superior ao Modelo $2\left[\Delta \chi^{2}(6)=108,26, p<0,001\right]$. Esse modelo bifatorial, com correlação livre entre os fatores e com seis itens saturando em ambos, também foi testado com o Grupo 2 (Modelo 9). Como para o Grupo 1, esse modelo foi o que apresentou os melhores índices de bondade de ajuste e foi estatisticamente superior ao Modelo $7\left[\Delta \chi^{2}(6)=108,26, p<0,001\right]$.

Na Tabela 2 são apresentados os 30 itens da escala e suas respectivas saturações (Lambdas) nos fatores para ambos os grupos. Note-se que para o Grupo 1 apenas os itens 2 e 3 não apresentaram saturações estatisticamente diferentes de zero $(t<1,96, p>0,05)$ e os fatores apresentaram uma correlação positiva e estatisticamente diferente de zero $(\phi=0,21, p<0,05)$. Cinco itens não apresentaram saturações estatis- ticamente diferentes de zero $(t<1,96, p>0,05)$ para 0 Grupo 2, os itens 2, 10, 17 e 30 para o fator Independente e o item 1 para o Interdependente. Para esse grupo os fatores apresentaram uma correlação inversa, mas não estatisticamente diferente de zero $(\phi=-0,17, p>0,05)$.

Em resumo, os resultados confirmam a estrutura bifatorial da EAl, com as auto-imagens Independente e Interdependente. No entanto, embora o modelo ortogonal tenha apresentado os melhores parâmetros psicométricos em estudos com amostras estadunidenses, no Brasil foi o modelo de estimação livre que apresentou os melhores parâmetros. Ainda, alguns itens apresentaram saturações em ambos os fatores, e as correlações entre os fatores não foram semelhantes para o Grupo 1 e Grupo 2.

\section{Teste da Estrutura Fatorial Alternativa}

O segundo objetivo deste estudo foi verificar a possibilidade de uma estrutura com seis fatores latentes, como sugerido por Hardin et al. (2004). Nesse sentido, 
Tabela 2. Saturações (Lambda) da análise fatorial confirmatória do modelo bifatorial de estimação livre da EAl com Grupo 1 ( $n=327$ ) e Grupo $2(n=331)$.

\begin{tabular}{|c|c|c|c|c|}
\hline \multirow{2}{*}{ Itens da Escala de Auto-Imagem } & \multicolumn{2}{|c|}{ Grupo 1} & \multicolumn{2}{|c|}{ Grupo 2} \\
\hline & Ind. & Inter. & Ind. & Inter. \\
\hline 1. Gosto de ser único e diferente das outras pessoas em muitos aspectos.t & $0,29^{*}$ & $-0,37^{*}$ & $0,53^{*}$ & 0,04 \\
\hline 2. Sinto-me mais à vontade tratando alguém de você, ainda que seja muito mais velho que eu. $\dagger$ & 0,11 & & 0,23 & \\
\hline 3. Mesmo quando discordo fortemente dos membros do grupo, evito uma confrontação. & & 0,16 & & $0,42^{*}$ \\
\hline 4. Respeito as pessoas de autoridade com as quais mantenho interação. & & $0,33^{*}$ & & $0,18^{*}$ \\
\hline 5. Faço minhas próprias coisas, independentemente do que pensam as outras pessoas. $\dagger$ & $0,64^{*}$ & & $0,64^{*}$ & \\
\hline 6. Respeito as pessoas que são modestas. & & $0,23^{*}$ & & $0,34^{*}$ \\
\hline 7. Sinto que é importante para mim agir como uma pessoa independente. $\dagger$ & $0,49^{*}$ & & $0,33^{*}$ & \\
\hline 8. Sacrificarei meu próprio interesse em benefício do grupo em que estou. & & $0,76^{*}$ & & $0,80^{*}$ \\
\hline 9. Prefiro dizer "não" diretamente do que me arriscar a ser mal interpretado. $†$ & $0,45^{*}$ & & $0,33^{*}$ & \\
\hline 10. É importante para mim ter uma grande imaginação. $\dagger$ & $0,30^{*}$ & & 0,18 & \\
\hline $\begin{array}{l}\text { 11. Levaria em consideração o conselho dos meus pais a respeito dos meus estudos ou da minha } \\
\text { profissão. }\end{array}$ & & $0,47^{*}$ & & $0,42^{*}$ \\
\hline 12. Sinto que meu destino se mistura com o destino daqueles que me rodeiam. & & $0,45^{*}$ & & $0,57^{*}$ \\
\hline 13. Prefiro ser direto e claro quando lido com pessoas que pouco conheço. $†$ & $0,45^{*}$ & & $0,40^{*}$ & \\
\hline 14. Sinto-me à vontade quando colaboro com os demais. & & $0,30^{*}$ & & $0,17^{*}$ \\
\hline 15. Sinto-me à vontade se sou indicado para receber um elogio ou um prêmio. $†$ & $0,43^{*}$ & & $0,52^{*}$ & \\
\hline 16. Se meu irmão ou minha irmã fracassasse, me sentiria responsável. & & $0,56^{*}$ & & $0,52^{*}$ \\
\hline $\begin{array}{l}\text { 17. Freqüentemente tenho a sensação de que minha relação com as outras pessoas é mais importante } \\
\text { do que minhas próprias realizações. }\end{array}$ & $-0,61^{*}$ & $0,72^{*}$ & $-0,05$ & $0,77^{*}$ \\
\hline 18. Dirigir-me às outras pessoas em uma aula (ou uma reunião) não é um problema para mim. † & $0,27^{*}$ & & $0,66^{*}$ & \\
\hline 19. Ofereceria meu lugar em um ônibus ao meu professor (ou meu chefe). & & $0,44^{*}$ & & $0,49^{*}$ \\
\hline 20. Comporto-me da mesma maneira esteja com quem estiver. $\dagger$ & $0,58^{*}$ & $0.90^{*}$ & $0,48^{*}$ & \\
\hline 21. Minha felicidade depende da felicidade dos que me rodeiam. & & & & $0,10^{\prime \prime}$ \\
\hline 22. Valorizo estar bem de saúde acima de tudo. $\dagger$ & $0,68^{*}$ & & $0,34^{*}$ & \\
\hline 23. Permanecerei em um grupo que necessita de mim, ainda que não esteja feliz com ele. & & $0,41^{*}$ & & $0,77^{*}$ \\
\hline $\begin{array}{l}\text { 24. Tento fazer o que é melhor para mim, independentemente de como poderia afetar as outras } \\
\text { pessoas. }+\end{array}$ & $0,45^{*}$ & $-0,50^{*}$ & $0,37^{*}$ & $-0,33^{*}$ \\
\hline 25. Ser capaz de me cuidar é um interesse principal em minha vida. $†$ & $0,63^{*}$ & & $0,58^{*}$ & \\
\hline 26. É importante para mim respeitar as decisões tomadas pelo grupo. & & $0,40^{*}$ & & $0,36^{*}$ \\
\hline 27. É muito importante para mim que minha identidade pessoal não dependa das outras pessoas. $†$ & $0,43^{*}$ & & $0,36^{*}$ & \\
\hline 28. É importante para mim manter a harmonia dentro do meu grupo. & $0,26^{*}$ & $0,26^{*}$ & $0,13^{*}$ & $0,24^{*}$ \\
\hline 29. Comporto-me da mesma maneira em casa e na escola (ou no trabalho). $\dagger$ & $0,49^{*}$ & $0,33^{*}$ & $0,57^{*}$ & $0,28^{*}$ \\
\hline $\begin{array}{l}\text { 30. Geralmente faço o que as outras pessoas querem fazer, ainda quando gostaria de fazer algo } \\
\text { diferente. }\end{array}$ & $-0,49^{*}$ & $0,33^{*}$ & $-0,21$ & $0,45^{*}$ \\
\hline
\end{tabular}

Notas: Os itens 1, 17, 24, 28, 29 e 30 foram fixados para saturar nos dois fatores. Os itens 5, 7 e 24 (Independentes) e os itens 12, 14e 30 (Interdependentes) são os seis itens adicionais da EAl. * Saturação diferente de zero ( $\mathrm{t}>1,96, p<0,05)$. † Itens especificados por Singelis (1994) como da auto-imagem Independente; Ind= independente; Inter=interdependente.

os itens da EAl foram especificados para apresentar seis fatores: Autonomia/Assertividade (itens 2, 3, 9, 13,15, 18 e 30), Individualismo (itens 1,5,6, 7, 10 e 27), Consistência Comportamental (itens 20 e 29), Primazia do Eu (itens

28422,24 e 25), Estima pelo Grupo (itens 3, 4, 11, 12, 14, 19, 23,
26, 28 e 30), e, por fim, Interdependência Relacional (itens 8, 16, 17 e 21) (E. E. Hardin, comunicação pessoal, 15 de julho de 2004).

Inicialmente esse modelo de seis fatores foi testado com os dois grupos, formando um modelo 
grupo-fatorial (-group-factor model, Rindskopf \& Rose, 1988, Figura 1c). Esse modelo indicou índices satisfatórios de bondade de ajuste para ambos os grupos: Grupo 1 $\left(\chi^{2}(388)=745,86 ; \chi^{2} / \mathrm{g} . \mathrm{I}=1,92 ; \mathrm{GFI}=0,87 ; \mathrm{AGFI}=0,84 ;\right.$ RMSEA $=0,053 ; 90 \%$ RMSEA $=0,047,0,059 ;$ SRMR $=0,071) \mathrm{e}$ Grupo $2\left(\chi^{2}(388)=796,19 ; \chi^{2} / \mathrm{g} . \mathrm{I} .=2,05 ; \mathrm{GFI}=0,86 ; \mathrm{AGFI}=\right.$ 0,83; RMSEA =0,056; 90\%RMSEA=0,051,0,062; SRMR= 0,069). Para o Grupo 1 apenas os itens 2 e 6 não apresentaram saturações estatisticamente diferentes de zero ( $t<1,96, p>0,05)$. Os itens 2 e 6 também não apresentaram saturações estatisticamente diferentes de zero para o Grupo 2, além do item 3 (no fator Autonomia/ Assertividade).

Para comprovar a estrutura fatorial com seis fatores, uma nova AFC foi realizada, dessa vez com toda a amostra ( $n=658$ ) e testando um modelo hierárquico, ou modelo fatorial de segunda ordem (Rindskopf \& Rose, 1988, Figura 1b). Nesse modelo, os seis fatores descritos anteriormente foram fixados como fatores de primeira ordem e as auto-imagens Independente e Interdependente como fatores de segunda ordem. Seguindo os procedimentos de Hardin et al. (2004), a saturação de um item por fator de primeira ordem (itens 2, 1, 20,22,03 e 08) e as variâncias dos fatores de segunda ordem foram fixadas em 1,00, e as variâncias de erros do modelo (error variances) de um par dos fatores de primeira ordem (Consistência Comportamental com Primazia do Eu, e Estima pelo Grupo com Interdependência Relacional) foram fixadas para serem iguais. Novamente, os índices de bondade de ajuste foram satisfatórios $\left(\chi^{2}(398)=1163,99 ; \chi^{2} / g . l=2,92\right.$; $\mathrm{GFI}=0,89 ; \mathrm{AGF}=0,88 ; \mathrm{RMSEA}=0,054 ; 90 \% \mathrm{RMSEA}=0,051$, 0,058; SRMR= 0,064). Esse modelo hierárquico com as respectivas saturações estandardizadas é apresentado na Figura 2.

Os coeficientes de consistência interna dos seis fatores foram: Autonomia/Assertividade $(\alpha=0,30)$, Individualismo $(\alpha=0,42)$, Consistência Comportamental $(\alpha=0,60)$, Primazia do Eu $(\alpha=0,39)$, Estima pelo Grupo $(\alpha=0,46)$ e Interdependência Relacional $(\alpha=0,54)$. Esses valores foram inferiores aos encontrados por Hardin et al. (2004). Todavia, o teste da igualdade dos coeficientes (van de Vijver \& Leung, 1997, p.60) indicou que os coeficientes de consistência interna encontrados no presente estudo não foram estatisticamente inferiores aos encontrados com a amostra estadunidense.
Resumidamente, os resultados comprovaram a adequação da estrutura com seis fatores subjacentes aos itens da EAI. Essa estrutura fatorial alternativa apresentou índices de bondade de ajuste adequados para os Grupos 1 e 2. Um modelo fatorial hierárquico, com seis fatores de primeira ordem e dois de segunda, também apresentou índices de bondade de ajuste adequados para toda a amostra.

\section{Teste da Utilidade da Estrutura Fatorial Alternativa}

A utilidade da estrutura fatorial alternativa foi examinada através da validade de critério e convergente-discriminante. Com relação à validade de critério, uma ANOVA revelou certas diferenças quanto ao gênero. Embora não tenham sido encontradas diferenças significativas em relação à auto-imagem Independente [M=4,86 e 4,79, para homens e mulheres, respectivamente; $F(1,656)=1,72, p>0,05]$, e Interdependente $[M=4,64$ e 4,59, para homens e mulheres, respectivamente; $F(1,656)=1,43, p>0,05]$, o mesmo não ocorreu com os seis fatores. Dos seis, dois apresentaram diferenças significativas. Os homens $(M=5,50)$ apresentaram médias significativamente superiores às médias das mulheres $(M=5,36)$ no fator Individualismo, $F(1,656)=6,41, p<0,05$, e no fator Interdependência Interrelacional $(M=3,96$ e 3,75, para homens e mulheres, respectivamente), $F(1,656)=5,71, p<0,05$.

Para verificar a validade convergente-discriminante, as pontuações dos participantes nas subescalas Independente e Interdependente e nos seis fatores foram correlacionadas com os valores humanos básicos (Tabela 3). De uma forma geral os resultados corroboram os previamente encontrados (Milfont et al., 2002). Especificamente, a auto-imagem Independente apresentou correlações diretas e significativas $(p<0,001)$ com os Valores Pessoais $(r=0,37)$ e Centrais $(r=0,22)$, enquanto que a auto-imagem Interdependente apresentou correlação inversa e significativa com os Valores Pessoais $(r=-0,13, p<0,01)$ e direta e significativa com os Sociais $(r=0,31, p<0,001)$. Porém, contrariando resultados anteriores, a auto-imagem Independente não apresentou correlação significativa e positiva com os Valores Sociais ( $r=-0,06$, ns), e a Interdependente apresentou correlação direta e significativa com os Centrais $(r=0,10, p<0,05)$. 


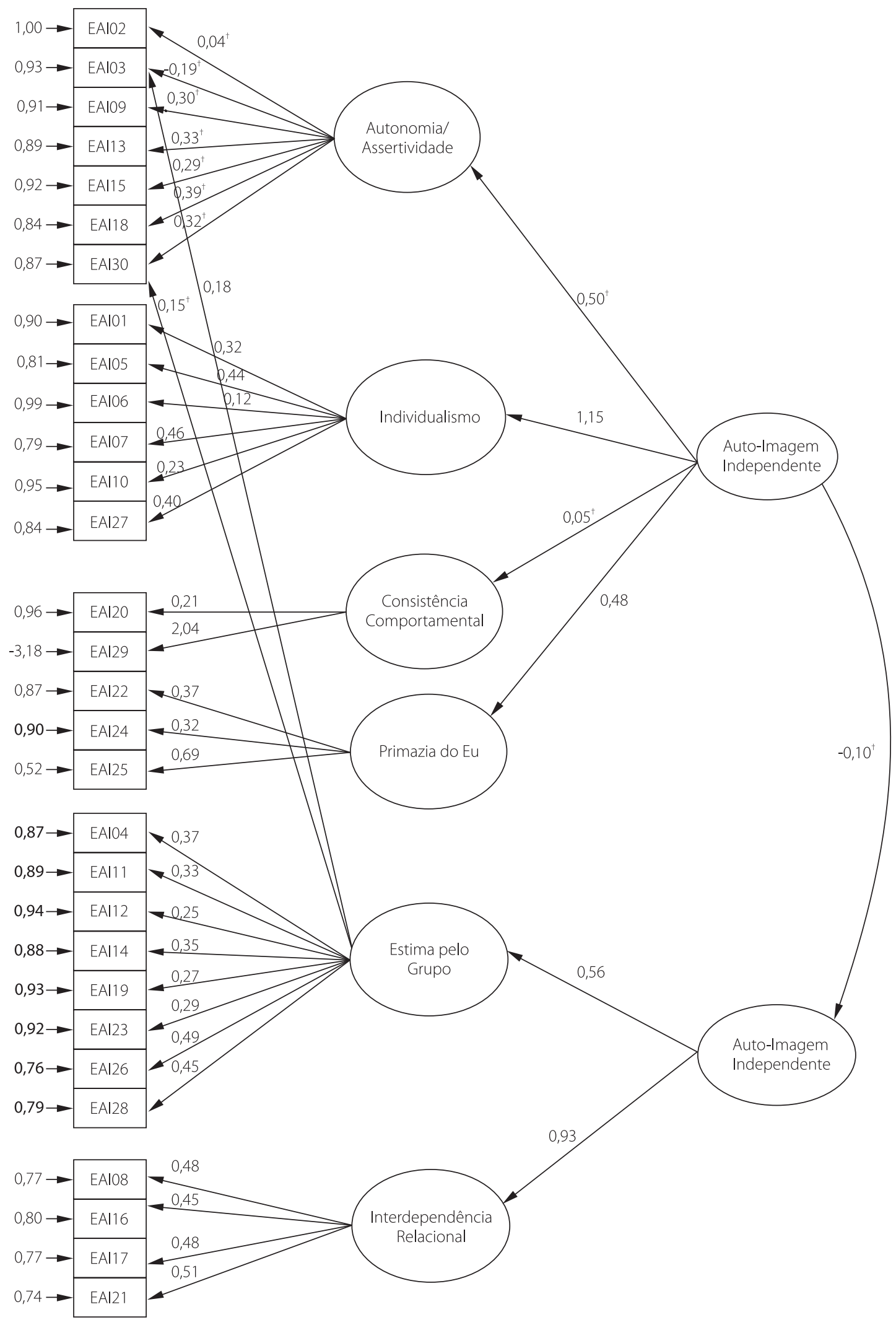

Figura 2. Solução estandartizada da análise fatorial confirmatória de segunda ordem da EAI ( $n=658)$.

Notas: Indices de bondade de ajuste do modelo: $\chi^{2}(398)=1163,99 ; \chi^{2} / \mathrm{g} . \mathrm{I} .=2,92 ; \mathrm{GFI}=0,89 ; \mathrm{AGFI}=0,88 ; \mathrm{RMSEA}=0,054 ; \mathrm{SRMR}=0,064$.

286

† Saturações não estatisticamente diferentes de zero $(\mathrm{t}<1,96, p 0,05)$. 
Tabela 3. Índices de consistência interna e correlações dos dois fatores originais da EAl com os seis novos fatores e valores humanos $(n=658)$.

\begin{tabular}{|c|c|c|c|c|c|c|c|c|c|c|c|c|c|c|}
\hline Subescala & $n$ & M & DP & 1 & 2 & 3 & 4 & 5 & 6 & 7 & 8 & 9 & 10 & 11 \\
\hline 1. Independente & 15 & 4,83 & 0,60 & $(0,50)$ & & & & & & & & & & \\
\hline 2. Interdependente & 15 & 4,61 & 0,60 & $-0,02$ & $(0,54)$ & & & & & & & & & \\
\hline $\begin{array}{l}\text { 3. Autonomia/ } \\
\text { Assertividade }\end{array}$ & 7 & 4,59 & 0,76 & $0,62^{* * *}$ & $-0,24^{* * *}$ & $(0,30)$ & & & & & & & & \\
\hline 4. Individualismo & 6 & 5,43 & 0,70 & $0,65^{* * *}$ & 0,02 & $0,27 * * *$ & $(0,42)$ & & & & & & & \\
\hline $\begin{array}{l}\text { 5. Consistência } \\
\text { Comportamental }\end{array}$ & 2 & 4,28 & 1,63 & $0,51^{* * *}$ & $0,10^{* *}$ & $0,12^{* * *}$ & $0,10^{*}$ & $(0,60)$ & & & & & & \\
\hline 6. Primazia do Eu & 3 & 4,73 & 1,01 & $0,56^{* * *}$ & $-0,09^{*}$ & $0,12^{* *}$ & $0,23^{* * *}$ & $0,10^{*}$ & $(0,39)$ & & & & & \\
\hline $\begin{array}{l}\text { 7. Estima pelo } \\
\text { Grupo }\end{array}$ & 10 & 4,80 & 0,60 & $-0,01$ & $0,88^{* * *}$ & $-0,31^{* * *}$ & $-0,06$ & $0,09^{*}$ & $-0,01$ & $(0,46)$ & & & & \\
\hline $\begin{array}{l}\text { 8. Interdependência } \\
\text { Relacional }\end{array}$ & 4 & 3,86 & 1,12 & $-0,04$ & $0,76^{* * *}$ & $-0,10$ & $-0,01$ & $0,08^{*}$ & $-0,16^{* * *}$ & $0,36^{* * *}$ & $(0,54)$ & & & \\
\hline 9. Valores Pessoais & 9 & 5,37 & 0,81 & $0,37^{* * *}$ & $-0,13^{* *}$ & $0,25^{* * *}$ & $0,32^{* * *}$ & $-0,05$ & $0,33^{* * *}$ & $-0,12^{* *}$ & $0,13^{* *}$ & $(0,58)$ & & \\
\hline 10. Valores Centrais & 7 & 6,15 & 0,62 & $0,22^{* * *}$ & $0,10^{*}$ & $0,10^{* *}$ & $0,15^{* * *}$ & 0,07 & $0,22^{* * *}$ & $0,11^{* *}$ & 0,02 & $0,35^{* * *}$ & $(0,46)$ & \\
\hline 11. Valores Sociais & 8 & 5,83 & 0,73 & $-0,06$ & $0,31 * * *$ & $-0,06$ & $-0,16^{* * *}$ & $0,09^{*}$ & 0,00 & $0,34^{* * *}$ & $0,17^{* * *}$ & $-0,07$ & $0,30^{* * *}$ & $(0,49)$ \\
\hline
\end{tabular}

Notas: Os valores na diagonal correspondem aos alfas de Cronbach das escalas. ${ }^{*} p<0,05 .{ }^{* *} p<0,01 .{ }^{* *} p<0,001$.

Com relação aos seis novos fatores, apenas três (Autonomia/Assertividade, Primazia do Eu e Estima pelo Grupo) seguiram os mesmos padrões de correlação das auto-imagens Independente e Interdependente. O fator Individualismo correlacionou-se inversa e significativamente com os Valores Sociais $(r=-0,16, p<0,001)$ e o fator Interdependência Relacional não correlacionou-se significativamente com os Centrais ( $r=0,02, n s)$. $O$ fator Consistência Comportamental foi o que apresentou a maior diferença nas correlações, com correlação inversa, mas não significativa, com os Valores Pessoais $(r=0,05, n s)$, correlação não significativa com os Valores Centrais $(r=0,07$, ns) e correlação direta e significativa com os Sociais $(r=0,09, p<0,05)$.

Em síntese, os resultados indicaram a validade do modelo fatorial alternativo da EAl. Os resultados da ANOVA e das correlações apontaram para uma maior utilidade desse modelo alternativo, quando comparado ao modelo bifatorial da EAI.

\section{Discussão}

Este estudo teve como objetivo examinar a validade de construto da Escala de Auto-Imagem (Singelis, 1994), utilizando sua versão completa e com uma amostra diferente da utilizada na sua validação ao contexto brasileiro (Gouveia et al., 2002a). Além disso, procurou testar o modelo fatorial alternativo com seis fatores (Hardin et al., 2004), analisando sua validade de critério e convergente-discriminante. Diante dos resultados apresentados, espera-se que tais objetivos tenham sido cumpridos.

Com relação à validade de construto, os resultados das AFC demonstraram que a versão completa da EAl (com 30 itens) apresentou uma estrutura bifatorial, confirmando o modelo de self dual proposto por Markus e Kitayama (1991) e verificado empiricamente por Singelis (1994) e Hardin et al. (2004). Todavia, não se comprovou uma ortogonalidade das subescalas de auto-imagem, como apontada anteriormente (Singelis, 1994; Singelis \& Brown, 1995). Os resultados encontrados sugerem correlações significativas, embora fracas, entre as duas auto-imagens. Como discutido adiante, esses resultados indicam que as auto-imagens apresentam diferentes significados no Brasil.

Ainda com relação à validade de construto, os coeficientes de consistência interna foram inferiores aos encontrados em estudos prévios. Considerando os 24 itens originais da EAl, Singelis (1994) encontrou valores de alfa de Cronbach próximos de 0,70 para as autoimagens Independente e Interdependente, enquanto que Gouveia et al. (2002a) encontraram valores próximos de 0,50 . Com relação à versão completa, Hardin et al. (2004) também encontraram valores próximos de 0,70, enquanto que neste estudo os valores foram próximos de 0,50. Assim, pode-se supor que os parâmetros psicométricos da versão em Português da EAI são inferiores aos da versão original em Inglês. 
Segundo van de Vijver e Hambleton (1997), três tipos de erros podem ocorrer na utilização de instrumentos traduzidos para outra língua: erro de construto (relacionado à não equivalência do construto em questão para os dois grupos culturais), erro de método (pertinente a problemas na administração do instrumento) e erro de item (relacionado a problemas na tradução dos itens do instrumento). Os procedimentos de coleta de dados utilizados no Brasil foram os mesmos utilizados nas pesquisas internacionais, ou seja, administração de questionários anônimos respondidos individualmente pelos participantes em situação coletiva. A versão para o Português também seguiu procedimentos padrões para sua tradução e adaptação. Gouveia et al. (2002) traduziram e adaptaram a EAl através de um comitê bilíngüe, que é um procedimento recomendado em pesquisas transculturais (van de Vijver \& Leung, 1997). Essa versão em Português tem sido utilizada em diversos estudos (Gouveia et al., 1999; Milfont, 2001; Gouveia et al., 2002a; Gouveia et al., 2004). Dessa forma, apenas o erro de construto parece relevante.

Existe a possibilidade de que o construto investigado tenha significados diferentes no Brasil. Embora as auto-imagens tenham apresentado estrutura ortogonal em amostras norte-americanas e asiáticas, na cultura brasileira elas se revelaram como oblíquas. Mesmo consistente a idéia de um self dual, no Brasil não é tão clara a distinção entre um tipo de self e outro, ficando evidenciado com a quantidade de itens que saturaram em ambos fatores.

Os participantes da presente pesquisa parecem ser mais bem caracterizados como apresentando uma mistura dos dois tipos de self, aspecto que parece refletir bem o padrão de orientação dos brasileiros (Tamayo, 1997). Esse aspecto revela uma especificidade deste país, reforçando a idéia de que no contexto brasileiro predomina uma mistura de valores e orientações sociais (Gouveia, Andrade, Milfont, Queiroga \& Santos, 2003), sendo compatível assumir ao mesmo tempo aspectos de um self separado (independente) e conectado (interdependente) aos demais (Maia, Gouveia, Silva Filho, Milfont \& Lopes de Andrade, 2001).

Dessa forma, embora os resultados das AFC tenham indicado a validade de construto da EAI para o contexto brasileiro, as auto-imagens Independente e Interdependente não devem ser tomadas por ortogonais nesse contexto. Este estudo também tratou de uma estrutura fatorial alternativa, subjacente aos itens da EAI.

Seguindo os trabalhos empíricos sobre as diferentes tipologias no âmbito do individualismocoletivismo (Singelis et al., 1995; Triandis, 1995; Gouveia et al., 2002), Hardin et al. (2004) propuseram um modelo alternativo à estrutura bifatorial da EAl. Os índices de bondade de ajuste das AFC demonstraram que os seis fatores propostos por Hardin et al. também podem ser identificados no contexto brasileiro. Além disso, apesar de os alfas de Conbrach dos seis fatores $(0,30<\alpha<0,60$, $M=0,45)$ terem sido abaixo dos apresentados por Hardin et al. (2004) $(0,43<\alpha<0,64, M=0,57)$, os coeficientes não foram estatisticamente inferiores. Portanto, os resultados das AFC e dos índices de consistência interna do presente estudo também apoiam a validade de construto desse modelo alternativo para o contexto brasileiro (Watkins, 1989; van de Vijver \& Leung, 1997).

Além do respaldo empírico, a validade de construto do modelo alternativo também apresenta suporte teórico, pois alguns dos seis fatores são semelhantes aos fatores da Escala Multi-Fatorial de Individualismo e Coletivismo (EMIC) (Gouveia et al., 2002). Por exemplo, o fator Individualismo apresenta similaridades com o Individualismo Expressivo, no qual o desejo de ser único, ímpar e de ser reconhecido é ressaltado. Primazia do Eu, por sua vez, apresenta semelhanças com o Protoindividualismo, pois enfatiza a necessidade de sobrevivência e a capacidade de cuidar de si próprio.

Os resultados da Anova e das correlações com os valores básicos demonstram, ainda, a validade de critério e convergente-discriminante desta estrutura fatorial alternativa. Especificamente, os resultados indicaram que enquanto os grandes fatores Independente e Interdependente não discriminaram homens de mulheres, alguns dos fatores mais específicos o fizeram. Os homens apresentaram maiores escores do que as mulheres em Individualismo e Interdependência Relacional. Hardin et al. (2004) também verificaram que os homens apresentaram maiores escores nesse último fator. No entanto, os resultados do presente estudo, indicando que os homens apresentam ao mesmo tempo escores elevados em Individualismo e Interdependência Relacional, parecem contraditórios e precisam ser confirmados em pesquisas futuras. 
Em conjunto com os resultados apresentados por Hardin et al. (2004), este estudo demonstrou a necessidade de medir o construto dual de self por meio de distinções melhores e mais refinadas dentro de cada uma das auto-imagens Independente e Interdependente. Dessa forma, a estrutura com seis fatores subjacentes aos itens da EAI precisa ser considerada em futuras pesquisas, com a elaboração de itens específicos para cada fator, principalmente para o fator Consistência Comportamental, que é composto atualmente por apenas dois itens.

Para finalizar, um ponto importante a ser ressaltado diz respeito à nomenclatura que tem sido empregada para se referir aos construtos aqui tratados (Gouveia et al., 1999; Gouveia et al., 2002a; Gouveia et al., 2004). Mesmo sendo definido como aspectos da auto-imagem (Singelis et al., 1999), talvez tratar o self-construal proposto por Markus e Kitayama (1991) sob a terminologia de auto-imagem dificulte a intercomunicação entre os pesquisadores interessados na área. Por exemplo, as referências disponíveis no Index Psi sobre auto-imagem não contemplam o selfconstrual. Portanto, talvez fosse mais acertado, seguindo as publicações da Espanha (Espinosa, 2000; Gouveia \& Clemente, 1998), tratá-los como construção do eu.

\section{Referências}

Asch, S. (1952/1972). Psicologia social. São Paulo: Companhia Editorial Nacional.

Carmines, E.G., \& Mclver, J.D. (1981). Analyzing models with unobserved variables: Analysis of covariance structures. In G.W. Bohinstedt \& E.F. Borgatta (Eds.). Social measurement: Current Issues (pp.65-115). Beverly Hills, CA: Sage.

Cohen, A.B., \& Rozin, P. (2001). Religion and the morality of mentality. Journal of Personality and Social Psychology, 81 (4), 697-710

Cross, S.E., \& Madson, L. (1997). Models of the self: selfconstrual and gender. Psychological Bulletin, 122 (1), 5-37.

Espinosa, P. (2000). Razonamiento moraly conducta socialen el menor. Tese de doutorado não-publicada, Curso de Pós-Graduação em Psicologia, Universidade da Coruña. España.

Fabrigar, L.R., MacCallum, R.C., Wegener, D.T., \& Strahan, E.J. (1999). Evaluating the use of exploratory factor analysis in psychological research. Psychological Methods, 4 (3), 272-299.

Garson, G.D. (2003). PA 765 Statnotes: an online textbook. Available from: http://www2.chass.ncsu.edu/garson/ pa765/statnote.htm (Acess 2004 Jul 15).
Gouveia, V.V. (1998). La natureza de los valores descriptores del individualismo y del colectivismo: Una comparación intrae intercultural. Tese de doutorado não-publicada, Curso de Pós-Graduação em Psicologia Social, Universidade Complutence de Madri. España.

Gouveia, V.V., \& Clemente, M. (1998). La medida del individualismo y del colectivismo: la investigación en el campo de la psicología cultural. La Coruña: Servicio de Publicaciones.

Gouveia, V.V., Coelho, J.A.P.M., \& Vasconcelos, T.C. (1999). Auto-imagem e relações interpessoais: Sua variação intracultural. [Resumo]. Em Associação Brasileira de Psicologia Social, Resumos de comunicações científicas, $10^{\circ}$ Encontro Nacional da Associação Brasileira de Psicologia Social (p.86). São Paulo: Abrapso.

Gouveia, V.V., Singelis, T.M., \& Coelho, J.A.P.M. (2002a). Escala de auto-imagem: Comprovação da sua estrutura fatorial. Avaliação Psicológica, 1 (1), 49-59.

Gouveia, V.V., Andrade, J.M., Jesus, G.R., Meira, M., \& Soares, N.F. (2002b). Escala multi-fatorial de individualismo e coletivismo: Elaboração e validação de construto. Psicologia: Teoria e Pesquisa, 18 (2), 203-212.

Gouveia, V.V. (2003a). A natureza motivacional dos valores humanos: Evidências acerca de uma nova tipologia. Estudos de Psicologia, 8 (3), 431-443.

Gouveia, V.V., Andrade, J.M., Milfont, T.L., Queiroga, F., \& Santos, W.S. (2003b). Dimensões normativas do individualismo e coletivismo: É suficiente a dicotomia pessoal vs. social? Psicologia: Reflexão e Crítica, 16 (2), 223-234.

Gouveia, V.V., Singelis, T.M., Guerra, V.M., Santos, W.S., \& Vasconcelos, T.C. (2004). Auto-imagem e sentimento de constrangimento. Manuscrito submetido à publicação.

Hardin, E.E., Leong, F.T.L., \& Osipow, S.H. (2001). Cultural relativity in the conceptualization of career maturity. Journal of Vocational Behavior, 58 (1), 36-52.

Hardin, E.E., Leong, F.T.L., \& Bhagwat, A.A. (2004). Factor structure of the self-construal scale revisited: Implications for the multidimensionality of self-construal. Journal of Cross-Cultural Psychology, 35 (3), 327-345.

Hong, S., Malik, M.L., \& Lee, M.-K. (2003). Testing configural, metric, scalar, and latent mean invariance across genders in sociotropy and autonomy using a non-western sample. Educational and Psychological Measurement, 63 (4), 636-654.

Hu, L.-T., \& Bentler, P.M. (1999). Cutoff criteria for fit indexes in covariance structure analysis: Conventional criteria versus new alternatives. Structural Equation Modeling, 6 (1), 1-55.

Jöreskog, K.G., \& Sörbom, D. (1996). LISREL 8: User's reference guides. Chicago, IL: Scientific Software International.

Kagitçibasi, C. (1997). Individualism and collectivism. Em J.W. Berry, M.H. Segall, \& C. Kagitçibasi (Eds.). Handbook of cross-cultural psychology. Social behaviorand applications (2nd ed. v.3, pp.1-49). Boston: Allyn and Bacon.

Kuhn, M.H., \& McPartland, T.S. (1954). An empirical investigation of self-attitudes. American Sociological Review, 19 (1), 68-76. 
Laros, J.A. (2004). O uso da análise fatorial: al gumas diretrizes para pesquisadores. In L. Pasquali (Ed.). Análise Fatorial para pesquisadores (pp.147-170). Petrópolis: Vozes.

Maia, L.M. (2000). Prioridades valorativas e desenvolvimento moral: considerações acerca de uma teoria dos valores humanos. Dissertação de mestrado não-publicada, Curso de Pós-Graduação em Psicologia Social, Universidade Federal da Paraíba.

Maia, L., Gouveia, V.V., Silva Filho, S.B., Milfont, T.L., \& Lopes de Andrade, M.W.C. (2001). Prioridades valorativas e individualismo-coletivismo: Padrões de convergência. Psico, 32 (2), 7-24.

Markus, H.R., \& Kitayama, S. (1991). Culture and the self: Implications for cognition, emotion, and motivation. Psychological Review, 98 (2), 224-253.

Milfont, T.L. (2001). A intenção de contituir família: Suas bases normativas e relacionais. Dissertação de mestrado nãopublicada, Curso de Pós-Graduação em Psicologia Social, Universidade Federal da Paraíba.

Milfont, T.L., Gouveia, V.V., Chaves, S.S.S., Socorro, T.C., \& Queiroga, F. (2002). Valores humanos e auto-imagem: Padrões de convergência e evidências de especificidades. Psico, 33 (2), 427-446.

Milfont, T.L., Gouveia, V.V., \& Costa, J.B. (no prelo). Determinantes psicológicos da intenção de constituir família. Psicologia: Reflexão e Crítica.

Oishi, S., Schimmack, U., Diener, E., \& Suh, E.M. (1998). The measurement of values and individualism-collectivism. Personality and Social Psychology Bulletin, 24 (11), 1177-1189.

Ozawa, K., Crosby, M., \& Crosby, F. (1996). Individualism and resistance to affirmative action: A comparison of Japanese and American samples. Journal of Applied Social Psychology, 26 (13), 1138-1152.

Rhee, E., Uleman, J.S., \& Lee, H.K. (1996). Variations in collectivism and individualism by ingroup and culture: Confirmatory factor analysis. Journal of Personality and Social Psychology, 71 (5), 1037-1054.

Rindskopf, D., \& Rose, T. (1988). Some theory and applications of confirmatory second order factor analysis. Multivariate Behavioral Research, 23 (1), 51-67.

Sato, T., \& Cameron, J.E. (1999). The relationship between collective self-esteem and self-construal in Japan and Canada. Journal of Social Psychology, 139 (4), 426-435.

Schafer, J.L. (1997). Analysis of incomplete multivariate data. London: Chapman \& Hall.

Schafer, J.L., \& Graham, J.W. (2002). Missing data: our view of the state of the art. Psychological Methods, 7 (2), 147-177.

Sharkey, W.F., \& Singelis, T.M. (1995). Embarrassability and self-construal: A theoretical integration. Personality and Individual Differences, 19 (6), 919-926.

Singelis, T.M. (1994). The measurement of independent and interdependent self-construals. Personality and Social Psychology Bulletin, 20 (5), 580-591.
Singelis, T.M., Bond, M.H., Sharkey, W.F., \& Lai, S.Y. (1999). Unpackaging cuture's influence on self-esteem and embarrassability: The role of self-construal. Journal of Cross-Cultural Psychology, 30 (3), 315-341.

Singelis, T.M., \& Brown, W.J. (1995). Culture, self, and collectivist communication: Linking culture to individual behavior. Human Communication Research, 21 (3), 354-389.

Singelis, T.M., \& Sharkey, W.F. (1995). Culture, self-construal and embarrassability. Journal of Cross-Cultural Psychology, 26 (6), 622-644.

Singelis, T.M., Triandis, H.C., Bhawuk, D.S., \& Gelfrand, M. (1995). Horizontal and vertical dimensions of individualism and collectivism: A theoretical and measurement refinement. Cross-Cultural Research, 29 (3), 240-275.

Sinharay, S., Stern, H.S., \& Russell, D. (2001). The use of multiple imputation for the analysis of missing data. Psychological Methods, 6 (4), 317-329.

Smith, P.B., \& Bond, M.H. (1993). Social psychology across cultures: analysis and perspectives. New York: Harvester Wheatsheaf.

Schwartz, S.H. (1994). Are there universal aspects in the structure and contents of human values? Journal of Social Issues, 50 (4), 19-45.

Tamayo, A. (1981). EFA: escala fatorial de autoconceito. Arquivos Brasileiros de Psicologia, 33 (4), 87-102.

Tamayo, A. (1997). Os valores do brasileiro: uma década de pesquisa. Cadernos de Psicologia, 1 (1), 115-134.

Thompson, B. (2000). Ten commandments of structural equation modeling. In L.G. Grimm \& P.R. Yarnold (Eds.). Reading and understanding more multivariate statistics (pp. 261-284). Washington, DC: American Psychological Association.

Triandis, H.C. (1995). Individualism and collectivism. Boulder, CO: Westview Press.

van de Vijver, F.J., \& Hambleton, R.K. (1997). Translating tests: some practical guidelines. European Psychologist, 1 (2), 89-99.

van de Vijver, F.J., \& Leung, K. (1997). Methods and data analysis for cross-cultural research. Newbury Park, CA: Sage.

Watkins, D. (1989). The role of confirmatory factor analysis in cross-cultural research. International Journal of Psychology, 24 (6), 685-701.

West, S.G., Finch, J.F., \& Curran, P.J. (1995). Structural equation models with nonnormal variables: problems and remedies. In R.H. Hoyle (Ed.). Structural equation modeling: Concepts, issues and applications (pp.56-75). Newbury Park, CA: Sage.

Yamada, A.M., \& Singelis, T.M. (1999). Biculturalism and self-construal. International Journal of Intercultural Relations, 23 (5), 697-709.

Recebido para publicação em 19 de setembro de 2004 e aceito em 19 de maio de 2005. 